\title{
Key Performance Indicators for the Impact of Cognitive Assembly Planning on Ramp-Up Process
}

\author{
Christian Buescher, ${ }^{1}$ Eckart Hauck, ${ }^{2}$ \\ Daniel Schilberg, ${ }^{1}$ and Sabina Jeschke ${ }^{1}$ \\ ${ }^{1}$ Institute of Information Management in Mechanical Engineering IMA, RWTH Aachen University, \\ Dennewartstrasse 27, 52068 Aachen, Germany \\ ${ }^{2}$ Institute for Management Cybernetics e.V.(IfU), Dennewartstrasse 27, 52068 Aachen, Germany
}

Correspondence should be addressed to Christian Buescher,

christian.buescher@ima.rwth-aachen.de

Received 2 March 2012; Accepted 29 May 2012

Academic Editor: George Chryssolouris

Copyright (C) 2012 Christian Buescher et al. This is an open access article distributed under the Creative Commons Attribution License, which permits unrestricted use, distribution, and reproduction in any medium, provided the original work is properly cited.

\begin{abstract}
Within the ramp-up phase of highly automated assembly systems, the planning effort forms a large part of production costs. Due to shortening product lifecycles, changing customer demands, and therefore an increasing number of ramp-up processes, these costs even rise. So assembly systems should reduce these efforts and simultaneously be flexible for quick adaption to changes in products and their variants. A cognitive interaction system in the field of assembly planning systems is developed within the Cluster of Excellence "Integrative production technology for high-wage countries" at RWTH Aachen University which integrates several cognitive capabilities according to human cognition. This approach combines the advantages of automation with the flexibility of humans. In this paper the main principles of the system's core component-the cognitive control unit-are presented to underline its advantages with respect to traditional assembly systems. Based on this, the actual innovation of this paper is the development of key performance indicators. These refer to the ramp-up process as a main objective of such a system is to minimize the planning effort during ramp-up. The KPIs are also designed to show the impact on the main idea of the Cluster of Excellence in resolving the so-called Polylemma of Production.
\end{abstract}

\section{Introduction}

In this paper, a set of key performance indicators (KPIs) is discussed describing the impact of a cognitive interaction system on the ramp-up period of highly automated assembly systems. The basis is a cognitive interaction system which is designed within a project of the Cluster of Excellence "Integrative production technology for high-wage countries" at RWTH Aachen University with the objective to plan and control an assembly autonomously. 
The overall objective of the Cluster of Excellence is to ensure the competitive situation of high-wage countries like Germany with respect to high-tech products, particularly in the field of mechanical and plant engineering. Yet these countries are facing increasingly strong competition by low-wage countries. The solution hypothesis derived in the mentioned Cluster of Excellence is seen in the resolution of the so-called Polylemma of Production, for example, by improving the ramp-up process.

The contribution of the project "Cognitive Planning and Control System for Production" is the development of a cognitive interaction system. Cognitive interaction systems in general are characterised by two facts. On the one hand, they comprise cognitive capabilities as mentioned before, and, on the other hand, they feature an interaction between the technical system and human operators [1]. One of the major challenges of the Polylemma of Production is to increase the efficiency of planning and simultaneously utilise the value stream approach in the domain of assembly which comes along with an improvement of the ramp-up process. The main results are the implementation of a cognitive control unit (CCU) as the key component of the cognitive interaction system and the construction of an assembly cell on the technical side to practically test the functionality of the CCU.

In this context, assembly tasks are a big challenge for planning systems, especially considering uncertain constraints, as implied in this approach. As a result, classic planning approaches have shown to be of little use due to the huge computational complexity. By calculating the complex planning problems prior to the actual assembly, this problem can be bypassed-but current and temporary changes cannot be taken into account. That is why, in this project, a hybrid approach of pre- and replanning of assembly tasks is followed. While the CCU plans and controls the whole assembly process, the operator only executes assembly steps, which cannot be fulfilled by the robots and intervenes in case of emergency. In this way, the robot control, which is now based on human decision processes, will lead to a better understanding of the behaviour of the technical system and helps automating the ramp-up process.

The crucial point of the CCU is the reduction of planning costs compared to traditional automated assembly systems during ramp-up. This is reached by means of cognitive capabilities with simultaneously increasing the flexibility during the actual assembly process. To quantify this, a set of four KPIs is developed in this paper. These KPIs point out the influence of implementing a cognitive interaction system for assembly planning to the Polylemma of Production and particularly to the ramp-up period within a product development. The ramp-up process-period between completion of product design (pilot series release) and attainment of full capacity-is especially in automated production and assembly systems a planning- and cost-intensive phase. It includes the derivation of an assembly strategy, the design and possibly modelling of the assembly cell and the programming of the robots [2]. Here, the CCU makes an important contribution to minimize these expenses. The new KPIs therefore concentrate on the comparison of production systems with and without cognitive interaction systems while general performance measuring systems for the ramp-up itself already exist $[2,3]$.

The main contribution of this paper lies in the development and implementation of key performance indicators that can show the impact of cognitive interaction systems system on the ramp-up period of highly automated assembly systems using the example of the cognitive control unit developed within the Cluster of Excellence "Integrative production technology for high-wage countries" at RWTH Aachen University. The remainder of the paper is organized as follows: in the next section the ramp-up period in general and possible improvements of cognitive interaction systems within this step are described. In the first part 


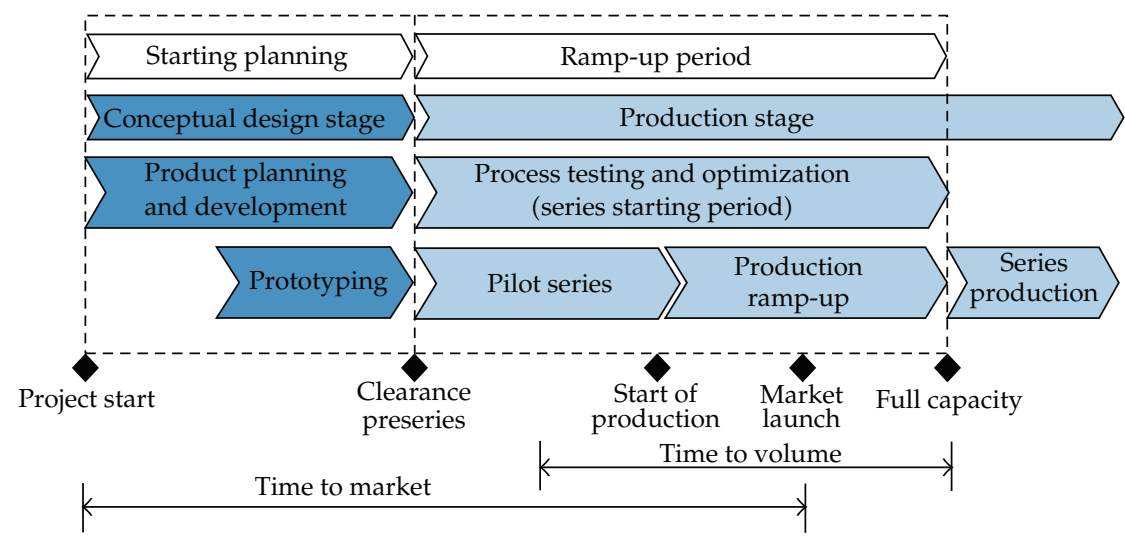

Figure 1: Ramp-up period within the product development process (according to [6-8]).

of Section 3, the idea of the Cluster of Excellence is introduced with a focus on the intended influence on production economics in high-wage countries centralised in the Polylemma of Production. Furthermore, the project, in which the presented cognitive interaction system is developed, is introduced. Section 4 is characterised by the presentation of the methodologies and strategies of the cognitive control unit. The new KPIs are presented and discussed in the Sections 5 and 6 . The paper concludes in the last section with future research directions.

\section{General Aspects of the Ramp-Up Period}

The product development process is divided into two stages (Figure 1):

(i) the conceptual design,

(ii) the production.

In the conceptual design stage, at first, the product planning is carried out in accordance with the market factors and the potential market. During the product planning, the process of the product development is already started and since these activities are iterative, they are grouped in a single phase. Furthermore, the joining ability of the single components is analysed in this phase. The product planning represents the transition between the idea for a product and its definition [4]. During the product planning and development, an initial prototype of the product is developed manually or by using a rapid prototyping technique. Thus, a first impression can be gained about the product and its haptic. In this phase, aspects of the assembly are already considered, since it is fatal to the assembly of a product if the individual components do not fit together [5].

As soon as the prototype has reached the desired developmental stage, the pilot series production starts. From this point on, different processes which form the ramp-up period flow simultaneously. The process testing and optimisation start with the pilot series. Within the literature, the pilot series is divided into a preseries, in which the rejection rates may be higher, and a pilot run, which represents the actual run-up to the production [9]. It ends with the start of the production that is with the actual ramp-up. This includes all activities 


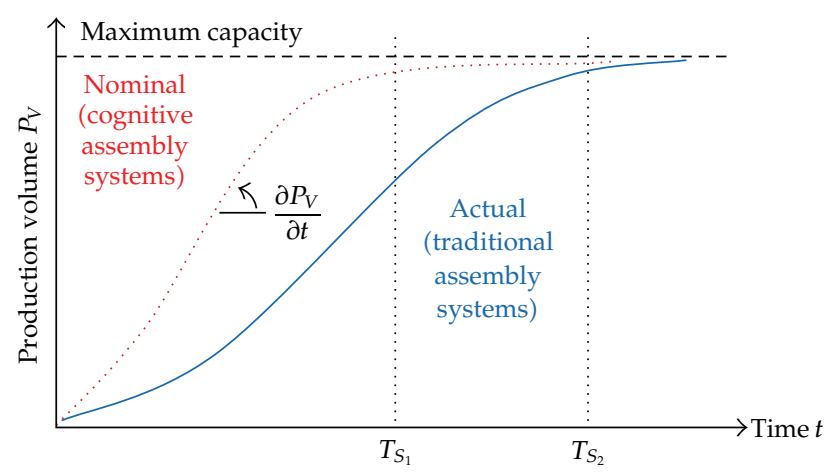

Figure 2: Characteristic of the ramp-up period with and without the use of cognitive interaction systems within the assembly system.

for planning, managing, and implementing the start-up, ranging from product and process development to mass production, including all upstream and downstream processes. The ramp-up phase involves the market launch and lasts up to the series production by reaching full capacity [6].

The installation of a cognitive interaction system has particularly influence on the ramp-up process for the assembly of a product. During the conceptual design stage, the effort for the planning of one assembly step in the manufacturing of a product is identical between a production that does not use systems with cognitive capabilities and a production that falls back to a cognitive interaction system. But starting the actual ramp-up with the pilot series, the programming and optimisation of a cognitive interaction system is more complex than the traditional assembly programming (e.g., through teaching of trajectories). Due to the higher flexibility of cognitive systems, the optimisation, however, can be made more quickly in a later series manufacturing. This advantage can only outweigh the disadvantages of increased complexity in the pilot series if multiple products are assembled on the same production system (utilise economies-of-scope) or a subsequent product change will be made.

Due to the fact that a cognitive interaction system is capable of responding to changing environmental conditions by varying its assembly sequence depending on the requirements by means of self-optimisation, several products can be manufactured in parallel on the same assembly cell [10]. In contrast, traditional programmed systems have to be readjusted for each product and assembly variation. Thereby, cognitive interaction systems can achieve a broadening of the product range without entailing significantly higher costs in the assembly.

This is especially reflected in the ramp-up period of a new product. Figure 2 shows the characteristics of a ramp-up process with the use of cognitive interaction systems within the assembly system and the corresponding phase of a production with traditional assembly systems. The ramp-up period of a production until reaching the capacity limit can be approximated by an $S$-curve (logistic function). The points in time $t_{S_{1}}$ and $t_{S_{2}}$ refer to the achievement of maximum series production capacity of each production system [11].

The $S$-curve is calculated according to the general equation [11]:

$$
f(t)=C * \frac{1}{1+e^{c *\left(t_{i}-t\right)}}
$$


with $f(t)$ being ramp-up function, $C$ being maximum capacity, $t$ being time, $t_{i}$ being time of the inflection point of the ramp-up function, and $c$ being constant.

This equation has a turning point on the site $t_{i}$, where the slope of the curve is maximal. At this point, the biggest gain in production volume per time unit is effected, that is, $\left.\left(\partial P_{V} / \partial t\right)\right|_{t=t_{i}}=$ max. In the progress of the curve, it is assumed that all optimisation steps within the production system, excluding the assembly step, are identical for both rampup curves. In a ramp-up process without the use of a cognitive interaction system, this slope matches the learning curve of operators who participate in the mounting process and optimise the assembly step. In a system containing a cognitive interaction system, this learning effect is enforced by the self-optimisation of the system during the process. That means that such a production system can reach the maximum capacity more quickly (see Figure 2, left curve).

To concretise these possible advantages of cognitive technical systems within the ramp-up period the next sections present a precise system with the CCU. First of all the idea of the Cluster of Excellence is introduced which builds the basis for the development of this system. Both descriptions are mandatory to follow the creation and discussion of the KPIs in Sections 5 and 6 in detail.

\section{Cluster of Excellence "Integrative Production Technology for High-Wage Countries"}

The aim of the Cluster of Excellence "Integrative production technology for high-wage countries" at RWTH Aachen University is to assure the production in high-wage countries by developing basics for sustainable production-scientific strategies and theories, as well as the necessity of technological approaches. To reach this objective, production in high-wage countries has to be regarded from different angles of visions and in an integrative manner [12]. Several institutes of RWTH Aachen University, which are dealing with the diversity of production engineering, are united under the Cluster of Excellence.

\subsection{The Polylemma of Production}

Competitive production engineering is particularly important for high-wage countries such as Germany as other competing countries, for example, in Eastern Europe or Asia, have much lower-factor costs. The influence of these low-wage countries has a tremendous impact on high-wage countries' economy due to all globalisation tendencies. Two dimensions can be identified within the competition between production companies in high-wage and lowwage countries: the production-orientated economy and the planning-orientated economy [13]. The global production industry is confronted with both dichotomies.

Due to low productive factor cost, low-wage countries' production can compensate possible economic disadvantages such as long process times, factor consumption, and process mastering. They predominantly focus on the utilisation of volume effects in production [12]. In contrast, companies in high-wage countries try to exploit the economies of scale by the usage of relatively expensive productivity factors. However, while these disadvantages of relatively high unit costs emerge more and more, companies concentrate on customising, fast adaptions to market needs, and the usage of synergy effects within the production of related portfolios (economies of scope). In addition, the share of production within the value chain decreases, which in turn leads to a decrease of the realisable economies of scale. 


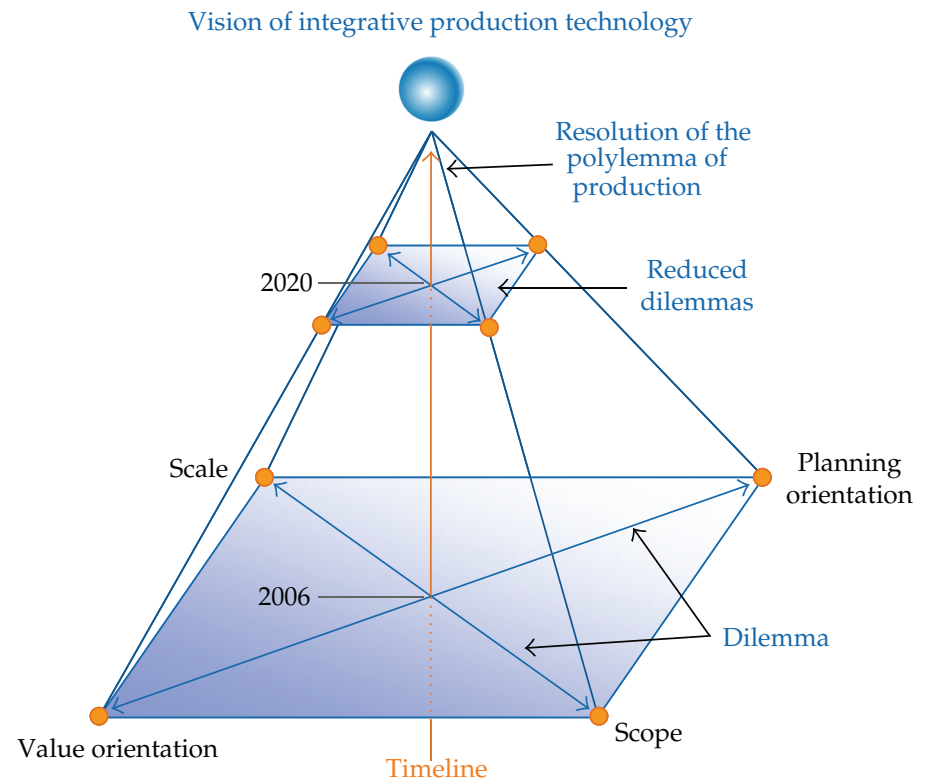

Figure 3: The Polylemma of Production ([13]).

Furthermore, the escape into sophisticated niche markets is typically not promising [12]. In general, companies in high-wage countries have to position themselves in-between scale and scope [13].

An additional competitive disadvantage for high-wage countries emerges on the planning-oriented economy. Companies often try to optimise processes with sophisticated, investment-intensive planning methods, and production systems since processes and production systems do not reach their limit of optimal operating points. This includes a complex ramp-up management to run production close to these optimal operating points. In contrast, companies in low-wage countries implement primarily simple, robust value-streamoriented process chains and do not concentrate on higher planning activities [12].

The dichotomy scale versus scope on the one hand and the dichotomy value versus planning orientation on the other hand are the dilemmas, production industry, especially in high-wage countries, has to face. These dichotomies span the so-called Polylemma of Production as shown in Figure 3 [13].

A better positioning within this Polylemma of Production is no longer sufficient to achieve sustainable competition advantages for the production in high-wage countries. Therefore the aim of the Cluster of Excellence is to possibly resolve both dichotomies by extending product variability and quality while simultaneously producing at series manufacturing costs [13].

\subsection{The Project "Cognitive Planning and Control System for Production"}

The challenge of the typical advanced rationalisation of traditional production systems and processes in high-wage countries is the implementation of value stream-orientated 
approaches with simultaneously increasing planning efficiency. To face this problem, the capability of self-optimisation is required. Self-optimisation allows an optimisation of real production processes without raising preceding planning costs and so optimizing the ramp-up process [10]. Therefore, within the domain "Self-Optimising Production Systems" of the mentioned Cluster of Excellence the focus lies on methods and technologies to enhance cognitive capabilities of production systems as the basis for self-optimisation. New perspectives of manufacturing and assembly systems are opened up by the application of existing knowledge to similar situations or new production cases as the core of self-optimisation [1].

The technological basis of the promising approach to reduce previous planning efforts by developing a production system that is able to autonomously plan the production during a running process and that could autonomously react to changes in customer demands is a novel architecture of the production cell's numerical control based on a cognitive architecture [14].

A cognitive interaction system with a cognitive planning and control unit (CCU) is developed within the project "Cognitive Planning and Control System for Production" to aim at the automation of the assembly planning process. The basic set-up of the CCU is founded on the architectures that illustrate human cognition. Therefore, a modularly assembled cognitive architecture for a production technology environment including a module to store knowledge and a human-machine interface is developed as a framework for the implementation of cognitive capabilities [15]. According to human cognition, these artificial cognitive capabilities are perception, reasoning, remembering, planning, decision making, learning, and action [16].

The approach is discussed as part of a scenario, which contains an assembly cell with two robots (Figure 4): one (Robot 2) is only controlled by the CCU [15, 17]; the other one (Robot 1) delivers separate parts for the final product in random sequence to a circulating conveyor belt. The CCU decides whether to pick up the delivered parts or to refuse them. In case that a part is picked up, it will be put into a buffer area or into the assembly area for immediate use. The practical assembly scenario, which also forms the basis of this paper, is a tower of four different coloured Lego bricks (see Figure 6).

This scenario bases on a random block delivery, which is a specialty of this approach and emphasises the flexibility of such a cognitive interaction system. Handling this kind of uncertainty in the system is a novelty. As a result, extensive material supply timing and planning, as it has to be done, for example, with Just in Sequence, is not necessary any more [18]. Instead the material storing has to be focused but which being cost-intensive than the whole planning problem [19]. Due to this approach, future states of the system cannot be predicted. The CCU is therefore facing a nondeterministic planning problem requiring either an online replanning during the assembly whenever a not expected event occurs or a plan in advance for all possible delivery sequences. Each of these strategies results in extensive computations, which lead either to slow responses during the assembly or an unacceptable amount of preplanning. Therefore, a hybrid approach is followed, which bases on state graphs [14].

The project's focus lies on evaluating the concept and methodology of this novel automation approach. To understand the basic ideas of the CCU, the next section will present the underlying assembly strategy including the determinations on which the assembly rules of the CCU are built. 


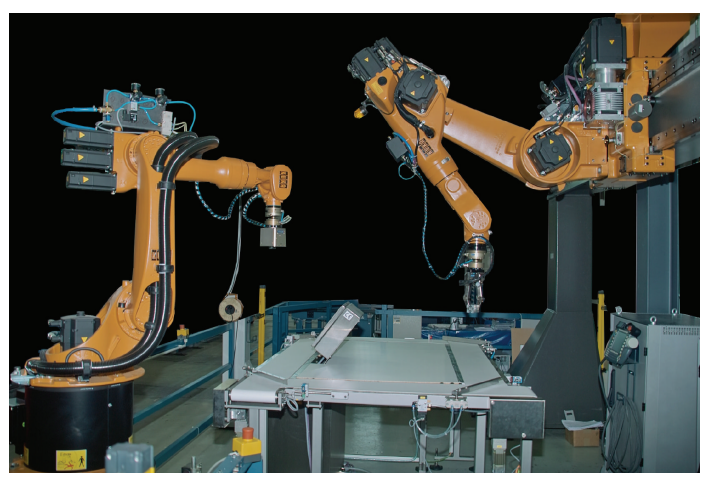

(a)

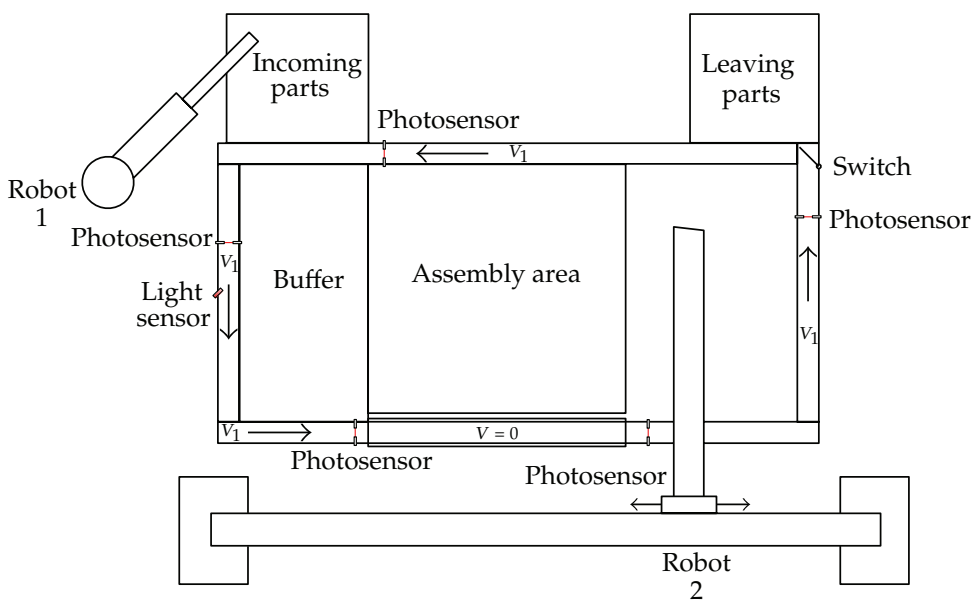

(b)

Figure 4: Photo and diagram of the robot cell.

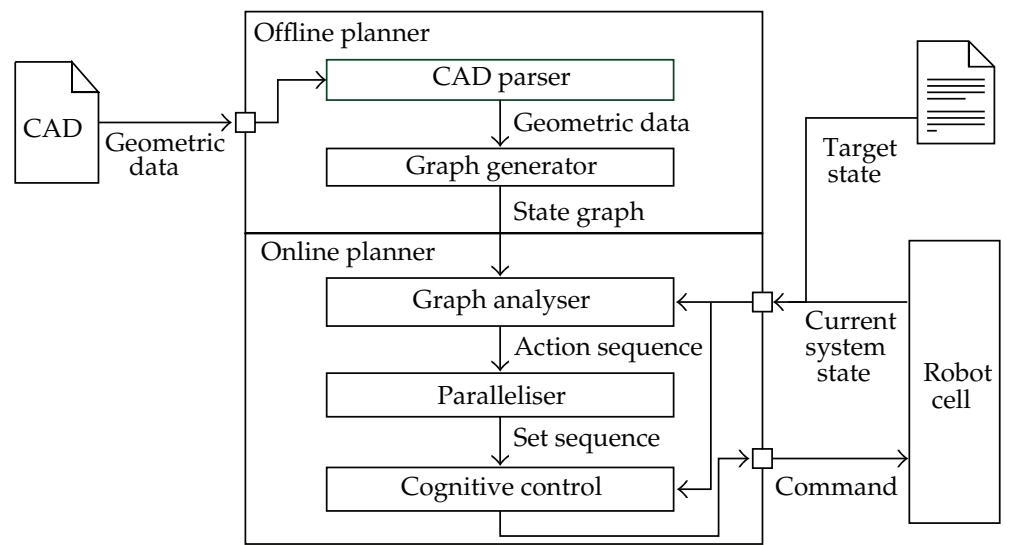

Figure 5: Hybrid approach of the CCU. 


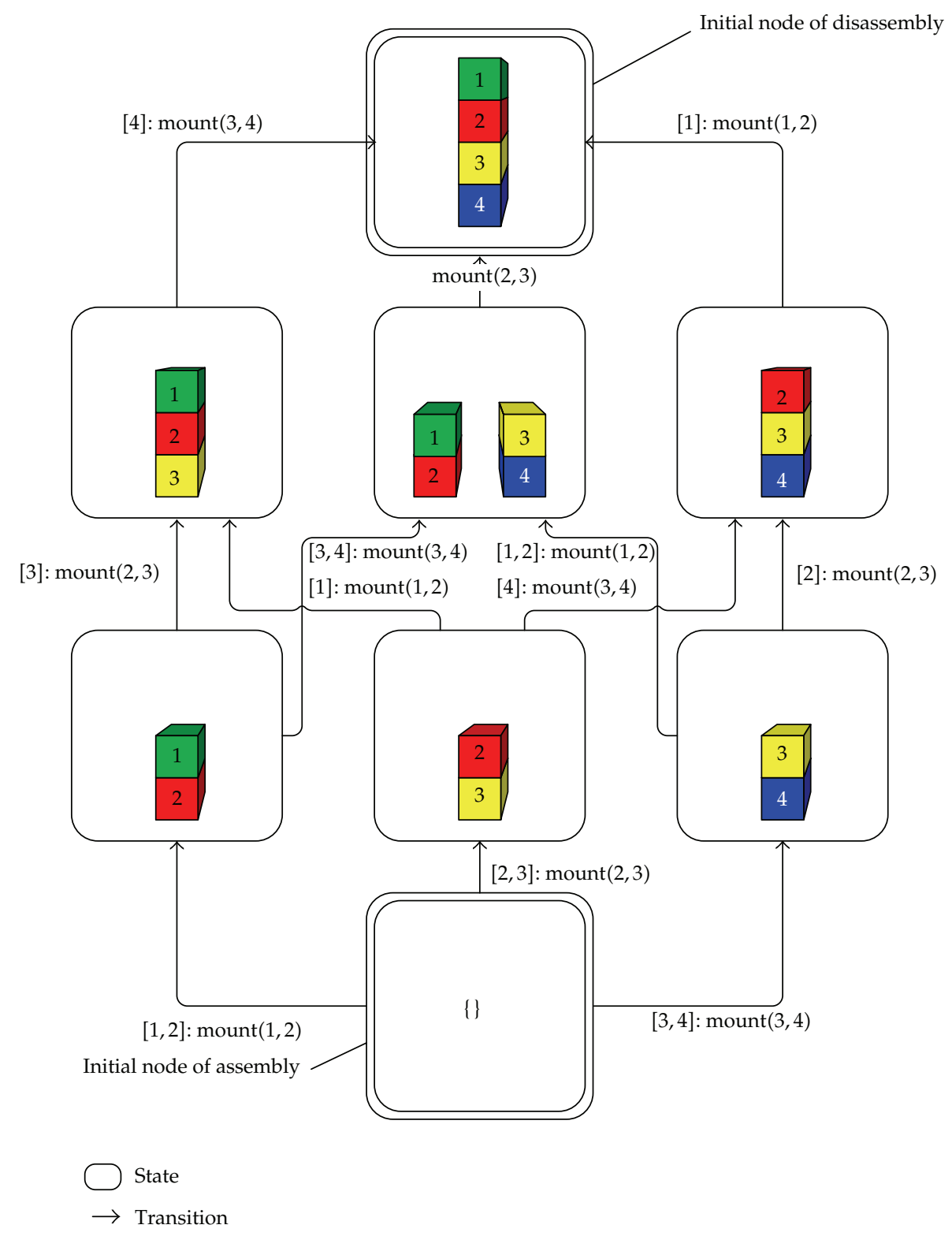

Figure 6: Example of a state graph representation.

\section{The Cognitive Control Unit}

The main idea of the cognitive control unit is to autonomously plan and control the assembly of a product solely by its CAD description and so help improve the ramp-up. Hence, it will be possible to decrease the planning effort in advance and to increase the flexibility of manufacturing and assembly systems [15]. Therefore several different approaches, which are suitable for the application on planning problems, are of great interest in the field of artificial intelligence. While generic planners like the ones by Hoffmann [20], Castellini et al. [21], 
and Hoffmann and Brafman [22] are not able to compute any solution within an acceptable time in the field of assembly planning concerning geometrical analysis, other planners are especially designed for assembly planning, for example, the widely used Archimedes System [23]. To find optimal plans, it uses AND/OR-graphs and an "assembly by disassembly" strategy. The approach of Thomas follows the same strategy, but uses only geometric information of the final product as input [24]. Nevertheless, both approaches are not adequate enough to deal with uncertainty. Another system developed by Zaeh and Wiesbeck [25] follows an approach which is similar to the CCU apart from the fact that it only plans and does not control the assembly. In this field, the CCU is a sophisticated system on the way to self-optimisation.

The CCU is able to take over tasks from an operator, for example, repetitive, dangerous and not-too-complex operations, as it is capable to process procedural knowledge encoded in production rules and to control multiple robots. As knowledge-based behaviour as well as skill-based behaviour cannot be modelled and simulated by the CCU, it will cooperate with the operator on a rule-based level of cognitive control [14, 26]. The task of the CCU consists of the planning and the controlling of the assembly of a product that is described by its CAD data while the assembly actions are executed by the assembly robots. After receiving an accordant description entered by a human operator, the system plans and executes the assembly autonomously by means of a hybrid planner. With regard to the cooperation between the $\mathrm{CCU}$ and an operator it is crucial that the human operator understands the assembly plan developed by the CCU. Furthermore, a robot control which is based on human decision processes will lead to a better understanding regarding the behaviour of the technical system which is referred to as cognitive compatibility [1].

\subsection{Hybrid Planner}

The planning process of the CCU is separated into two assembly parts to allow fast reaction times: the Offline Planner, executed prior to the assembly, and the Online Planner, executed in a loop during the assembly (Figure 5). The Offline Planner allows computation times of up to several hours. Its task is to precalculate all feasible assembly sequences-from the single parts to the desired product. The output is a graph representing these sequences. This graph is transmitted to the Online Planner whose computation time must not exceed several seconds. Its task is to map repeatedly the current system state to a state contained in the graph during assembly. In a further step, the Online Planner extracts an assembly sequence that transforms the latest state into a goal state containing the finished product. Thus, the proposed procedure follows a hybrid approach [14].

A solution space for the assembly sequence planning problem is derived during the offline planning phase. As mentioned above, an "assembly by disassembly" strategy is applied to generate an assembly graph, which is first generated as an AND/OR-graph and which is then transformed into a state graph that can be efficiently interpreted during online planning [27]. Therefore, a description of the assembled product's geometry and its constituting parts, possibly enriched with additional mating directions or mating operation specifications, is used. The geometric data is read by the CCU from a CAD file. The main concept of this strategy is a recursive analysis of all possibilities of an assembly or subassembly [28]. Any assembly or subassembly is separated into two further subassemblies until only single parts remain. All related properties of the product's assembly are stored. 
Additionally, instances can be used to describe functional aspects. This will be relevant if additional data apart from the part geometries is taken into account by the assembly planner [24].

All data of the separation evaluators is stored in the state graph. Figure 6 shows the state graph of the used scenario. The information contains static operation costs and mating operation descriptions for each assembly that might be active during assembly. All transition steps are represented by edges enhanced with the named costs. Each state contains the passive assembly, to which other parts may be added, starting with the empty one at the bottom up to the final product at the top.

The Online Planner derives the assembly plan during the assembly process. It uses the state graph provided by the Offline Planner as well as information about the current robot cell's situation. This approach is similar to the system developed by [25], in which assembly task instructions for a human operator are reactively generated.

The Graph Analyser receives the generated state graph from the offline planning phase and the actual world state describing the current situation of the assembly. Afterwards, the graph analyser maps this world state onto the matching state contained in the state graph. If this node and the "goal-node" are identical, the assembly has been completed and the process ends. Otherwise, the state graph is updated. Dynamic costs in terms of the availability of necessary parts are assigned to the state graphs edges-in addition to the costs, which already have been assigned to the edges during offline planning [14]. After this procedure, the optimal path to the goal-node is calculated using the $\mathrm{A}^{*}$ search algorithm, which represents the optimal assembly plan for the given current situation [29]. This path is tested for parallelization and sent to the Cognitive Control component, which executes the assembly in a further step.

The Cognitive Control component receives the assembly sequence, triggers the accordant robot commands, and communicates with the human operator so that the latter can operate, for example, in case of unforeseen changes. This component is based on Soar, a cognitive framework for decision finding that aims on modelling the human decision process [30]. Soar contains several production rules which are stored in the knowledge base. Furthermore, human assembly strategies are developed and implemented in the component to generate a higher degree of machine transparency and to enhance cognitive compatibility [26]. Thus, this component implements the cognitive capability of decision making so that the CCU in general is able to optimise its performance according to different delivered target states.

In this section, the background of the cognitive interaction system with regard to the planning algorithm and the possibilities of decision making within the technical system was described. The next section points out how this approach can help to improve the ramp-up process by defining KPIs for cognitive interaction systems.

\section{Key Performance Indicators}

In order to measure the influence of a cognitive interaction system on the ramp-up process, four key performance indicators were developed. As described in the previous sections, the reduction of planning efforts prior to the assembly is a main objective of cognitive interaction systems like the CCU. This approach enables a faster ramp-up for assembly and thereby comprises at best an increase of production volume during this phase. This has on the one hand a positive effect on the validity of the data generated and beyond that on the quality 
of the final production process. In addition, the increased flexibility allows not only a static assembly strategy like traditional automated systems, but the possibility to act adaptively within the framework of the generated plan. The four KPIs which show these advantages within the triple constraint (cost, time and quality) are:

(i) $K_{\mathrm{PE}}$ : planning effort (Section 5.1),

(ii) $K_{\mathrm{APV}}$ : acceleration of production volume growth (Section 5.2),

(iii) $K_{\mathrm{IPV}}$ : increase of production volume (Section 5.3),

(iv) $K_{\mathrm{PQ}}$ : plan quality (Section 5.4).

The contribution to the ramp-up process and the Polylemma of Production technology is determined by the comparison of the KPIs with and without the use of a cognitive interaction system to control an assembly of components of simple geometry like the scenario described in Section 3.2 [11]. All KPIs are defined in a way that the larger the value, the more superior is the cognitive system compared to the traditional one. The turning point where both systems are equal is—depending on the context of the precise KPI— 0 or 1 .

\subsection{Planning Effort}

The first KPI, the planning effort, refers to the phase of mounting and initial programming of the cognitive interaction system within an assembly system. The initial filing and maintenance of the knowledge base in a cognitive interaction system represents significantly more work compared to programming a traditional assembly system, for example, by teaching the robot. This effort is too high for a production system that is designed only for one product since a traditional assembly system can be programmed very quickly for a specified manufacturing step and this programming has to be adjusted only marginally during production. However, if the assembly system needs to be able to assemble a wide range of products with small batches, a traditional assembly system has to be repeatedly reprogrammed and optimised. In contrast, an assembly system with a cognitive interaction system can be adapted with little effort on a new product.

The key performance indicator $K_{\mathrm{PE}}$ is based on Schilberg [31] and is calculated from the sum over $n$ different products to be assembled by the efforts of the programming of the system, respectively, the creation of the knowledge base and the optimisation of the assembly:

$$
K_{\mathrm{PE}}=1-\left(\frac{\sum_{i=1}^{n} \mathrm{PE}_{i_{\text {cognitive }}}}{\sum_{i=1}^{n} \mathrm{PE}_{i_{\text {traditional }}}}\right) .
$$

With $K_{\mathrm{PE}}$ being Key performance indicator of the planning effort, $\mathrm{PE}_{i_{\mathrm{cogn} n i t i v e}}$ being Planning effort of an assembly system with a cognitive interaction system, and $\mathrm{PE}_{i_{\text {traditional }}}$ being Planning effort of a traditional assembly system.

The interval in which the KPI ranges is $[-\infty,+1]$. The extreme value $-\infty$ of the interval will be reached if the planning effort for $\mathrm{PE}_{i_{\text {traditional }}}$ is arbitrarily small or if $\mathrm{PE}_{i_{\text {cognitive }}}$ is an arbitrary large value. The other extreme value of 1 will be reached if $P E_{i_{\text {cognitive }}}$ is 0 [11].

By the automated assembly planning within the cognitive interaction system, this system only has to be reprogrammed if the assembly of the product to be manufactured 


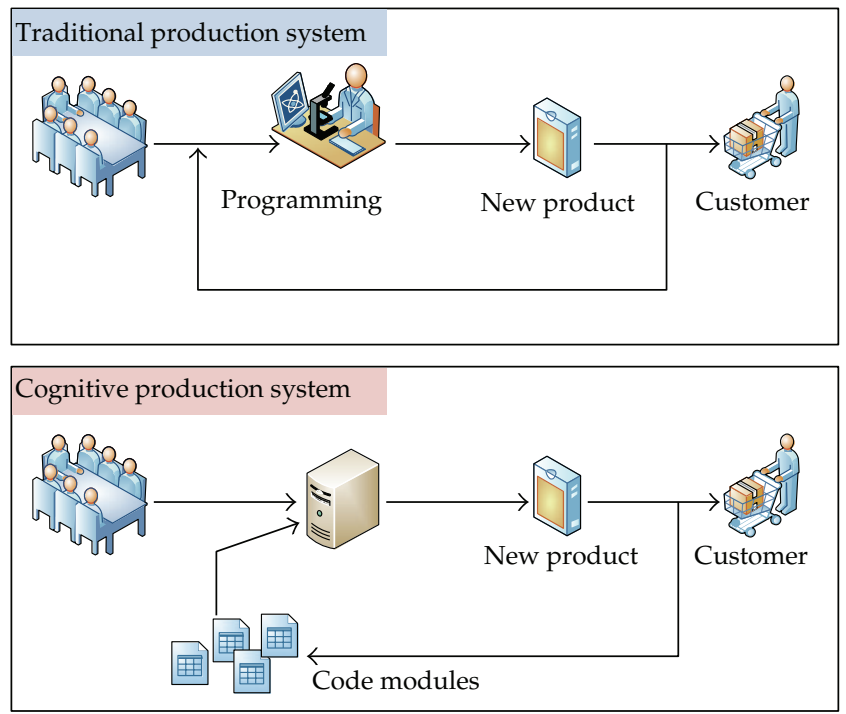

Figure 7: Work flow in the programming of the system for a traditional and a cognitive production system.

contains steps that were not previously stored in the cognitive interaction system (e.g., if a new tool is available, which results in new possible operations). By the independent planning of the assembly process, under constraints which have passed by the operator to the cognitive interaction system, no new operation sequences have to be programmed. The adaptive adjustment of the assembly sequence can even ensure an assembly with a notpreviously known component supply which is impossible in a traditional assembly system. When a new sequence of steps is to be executed, the assembly system has to be reprogrammed and optimised, which represents a significant amount of work. This does not allow flexible responds of the assembly system to changes in product manufacturing or in the assembly sequence. In Figure 7, an example of this comparison is illustrated.

\subsection{Acceleration of Production Volume Growth}

The second KPI, acceleration of production volume growth, is determined by comparing the maximum increase in production volume per time unit at the site $t_{i}$.

Therefore, the KPI is calculated as

$$
K_{\mathrm{APV}}=\left(\frac{\left.\left(\partial P_{V} / \partial t\right)\right|_{\text {cognitive }}}{\left.\left(\partial P_{V} / \partial t\right)\right|_{\text {traditional }}}\right)-1
$$

with $K_{\mathrm{APV}}$ being key performance indicator for acceleration of production volume growth, $\left.\left(\partial \mathrm{P}_{V} / \partial t\right)\right|_{\text {cognitive }}$ being slope at the inflection point of the production system with cognitive interaction systems, and $\left.\left(\partial P_{V} / \partial t\right)\right|_{\text {traditional }}$ being slope at the inflection point of the traditional production system.

By forming the quotient, the KPI ranges in the interval $[-1,+\infty]$. A value of -1 means that the production volume growth of a production system with cognitive interaction systems is 0 , so there is no production. The other extreme value means that the production 


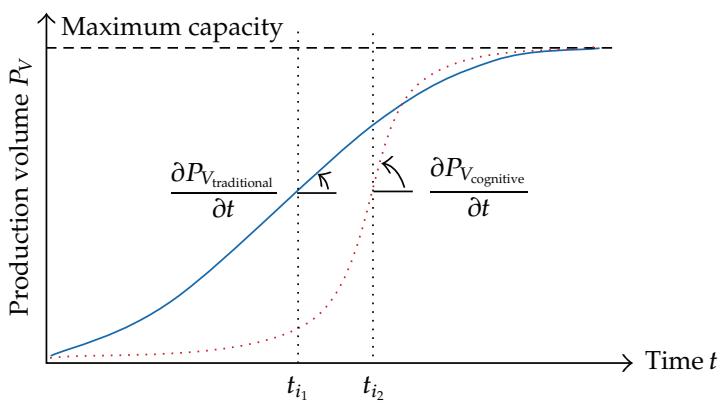

Figure 8: Example of a ramp-up process where the key performance indicator $K_{\mathrm{APV}}$ of a production system is higher, but the total production volume is smaller.

volume growth with a cognitive interaction system is arbitrarily large, respectively, and the production increase of a traditional production system is 0 . This value is never reached because it would mean a discontinuity in the S-curve, which has to be differentiable by definition [11].

In case of a congruence of the inflection points of the ramp-up function $f(t)$ for both production systems, it is sufficient to determine the key performance indicator $K_{\mathrm{APV}}$ if one production system dominates the other one. If the two inflection points do not match, it may happen that a production system, which has a steeper gradient but realises this at a significantly later time, possibly has a worse overall production volume. Figure 8 shows such an issue. The points in time $t_{i_{1}}$ and $t_{i_{2}}$ describe the inflection points with the maximum slope of the two curves.

\subsection{Increase of Production Volume}

In this case, a third KPI, namely the increase of total production volume during rampup, should be consulted. It is calculated by integrating over the starting function $f(t)$ in a given period. By the quotient, a direct comparison of production systems with and without cognitive interaction systems can be made.

Therefore, the KPI is calculated as:

$$
K_{\text {IPV }}=\frac{\int_{t_{0}}^{t_{s_{1}}} f_{\text {cognitive }}(t) d t}{\int_{t_{0}}^{t_{S_{2}}} f_{\text {traditional }}(t) d t}
$$

with $K_{\mathrm{IPV}}$ being key performance indicator of the increase of production volume, $t_{0}$ being start of production, and $t_{S_{i}}$ being time of series production with full capacity (see Figure 2).

Under the condition that the integral of $f(t)>0$, a cognitive system is superior to a traditional system if the KPI takes a value $>1$. The key performance indicator $K_{\mathrm{APV}}$ represents therefore the necessary condition for the superiority of a production system with cognitive interaction systems in the assembly, while the key performance indicator $K_{\mathrm{IPV}}$ is the sufficient condition for a real improvement [11]. This consideration is only meaningful if both times to volume differ from each other marginally. Otherwise the "faster" production system would always be preferred since time is often the critical variable in the ramp-up phase. 


\subsection{Plan Quality}

The fourth KPI is the plan quality, which is borrowed from one criterion for evaluation of planners on the International Planning Competition (IPC) [32]. It is determined from the number of assembly steps required to manufacture a product. In a traditional system, either the assembly sequence is fully optimised and programmed in advance or a heuristic-based optimisation is used by the employee during programming. Depending on the complexity of the product to be assembled, the optimal assembly sequence may not be found in a reasonable time. In the context of this scenario and further scenarios which were analysed within the project, the CCU is able to generate the entire assembly graph in the Offline Planner. At this point no heuristics have to be used but such applications are possible. Thus, this KPI is defined with regard to more complex products where heuristics are relevant.

As described before, in a cognitive interaction system, it may also be necessary to resort to a heuristic to solve the planning problem in a reasonable time in a corresponding product complexity. This planning can be continued during the production, which means that the cognitive interaction system starts with an assembly sequence that was found using a heuristic. Then during the process the system is able to derive a better assembly sequence in parallel by using relaxing heuristics and by conducting a broader search within the AND/OR-graph. If the number of components is below a threshold, all possible decompositions of the AND/OR-graph can be saved and a guaranteed optimal solution of the planning problem can be found [11]. Figure 9(a) shows the entire AND/OR-graph of a tower of four Lego bricks. In Figure 9(b), an assembly sequence using a heuristic is shown, in which only those nodes of the graph with the lowest costs are expanded.

The plan quality of the traditional and the cognitive assembly system is identical in this case, provided that the same resources for computing capacity and time exist. However, the cognitive assembly system is able to perform a broader search during the actual assembly and thus to create the optimal assembly plan (Figure 9(c)). In this example, this would mean a sum of costs of 40 instead of 50 although the first analysis step of the optimal solution is considerably larger than the one of the heuristic. The plan quality of a cognitive interaction system is thus at least as good as a traditional assembly system and is able to achieve a better plan quality which is expressed by a lower sum of costs in the assembly graph through an ongoing continuation of the planning.

The KPI is calculated as

$$
K_{\mathrm{PQ}}=\sum_{i=0}^{n} C_{i_{\text {traditional }}}-\sum_{i=0}^{m} C_{i_{\text {cognitive }}}
$$

with $K_{\mathrm{PQ}}$ being Key performance indicator of the plan quality, $n$ being number of assembly steps with traditional production system, $m$ being number of assembly steps with cognitive interaction system, $C_{i_{\text {traditional }}}$ being costs on optimal path with traditional production system, and $C_{i_{\text {cognitive }}}$ being costs on optimal path with cognitive interaction system.

Hence, $K_{\mathrm{PQ}}$ is never less than zero. In addition, the cognitive interaction system has the ability to adjust the assembly sequence depending on the availability of the single components. The optimal assembly sequence that was found during this process has been created under the terms of a deterministic supply of components respectively the availability of all components and assemblies required. With regard to the possibility of dynamic allocation of the new edge costs within the assembly graph in case of storage of all possible decompositions, the cognitive assembly system is able to adapt the assembly sequence 


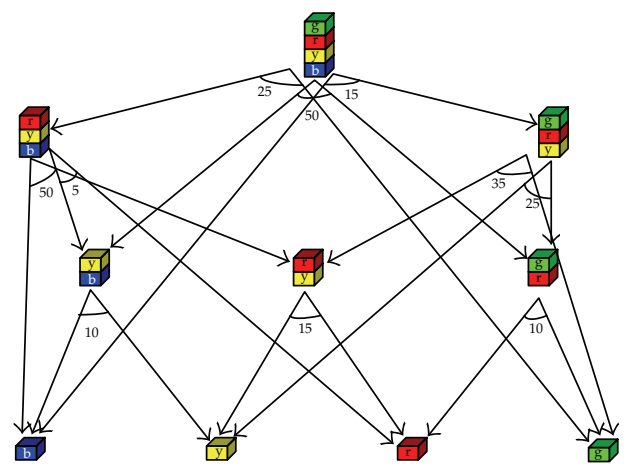

(a) Graph with all possible decompositions with 4 parts and the corresponding edge costs

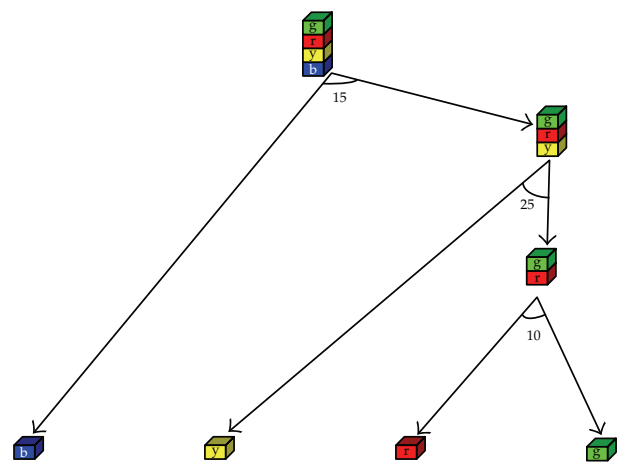

(b) Assembly sequence with the heuristic that only those nodes with the lowest costs are expanded

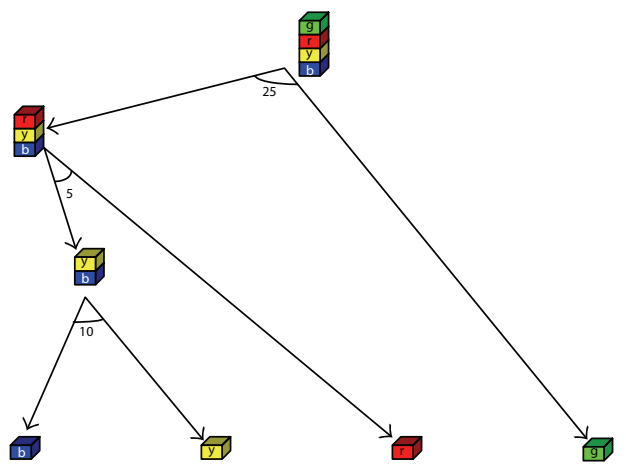

(c) Optimal assembly sequence with constant edge cost

Figure 9: Comparison of the plan quality.

dynamically and to ensure the optimal plan quality in a dynamic environment at any time during assembly.

\section{Discussion}

Against the background of the Polylemma of Production and improving the ramp-up process, the four KPIs presented in Section 5 are designed to identify the enhancements by using cognitive interaction systems with respect to traditional production systems. The main objective of the mentioned Cluster of Excellence is to develop new production theories and methods in order to resolve the Polylemma of Production inter alia by improving the ramp-up period and to measure the improvements reached by these innovations. Here, the KPIs provide a significant contribution. They cover the three dimensions costs $\left(K_{\mathrm{PE}}\right)$, time $\left(K_{\mathrm{APV}}\right.$ and $\left.K_{\mathrm{IPV}}\right)$ and quality $\left(K_{\mathrm{PQ}}\right)$, and thus represent any expected significant changes by comprising cognitive interaction systems like the CCU.

As pointed out in the last section, the indicators are defined in the following way: the larger the value is, the more superior is the cognitive system compared to the traditional one with a turning point by 0 or 1 . It is expected that the indicators $K_{\mathrm{APV}}$ and $K_{\mathrm{IPV}}$ are greater than the turning point. By automating the ramp-up process with the use of the CCU, it speeds up. 
The production can be ramped up more quickly so that $K_{\mathrm{APV}}$ increases. Furthermore, it is not necessary to carry out an intensive programming before the assembly cell can be launched. Instead, new production rules have to be implemented as well as to fill up the knowledge base and teach the system to optimize it. This can be done in parallel to the installation of the assembly cell so that the entire process is moved forward and $K_{\text {IPV }}$ increases as well. Thus, a certain dependency exists between the two indicators.

The planning efforts prior to the assembly change in their manner as indicated. Instead of programming the robots, a lot of work in educating the system has to be done when implementing a total new product and its assembly cell. In this case, at the present time, no conclusion can be drawn on the amount of total costs so that $K_{\mathrm{PE}}$ can either be positive or negative. The advantage of cognitive interaction systems consists in their flexibility to handle different variants of a product in one assembly cell at the same time. Here, at the best, no additional costs are generated so that $K_{\mathrm{PE}}$ is expected to be greater than zero. Since costs and time are interdependent, $K_{\mathrm{APV}}$ and $K_{\mathrm{IPV}}$ will increase as well.

The plan quality of a cognitive interaction system is, as pointed out in Section 5.4, at least as good as a traditional assembly system $\left(K_{\mathrm{PQ}} \geq 0\right)$. While in contemporary automated systems only the previously implemented assembly sequence is followed, the CCU and comparable systems can optimise themselves in the process due to their cognitive capabilities in terms of planning strategies and, if necessary, additionally used heuristics. This superiority increases the more the system is used as its database grows and as it learns from previous tasks for the generation of the plan, which results in a higher quality. The only way the plan quality of the traditional system can increase is a reprogramming (see Figure 7) due to human learning. This results in an increase of the other KPIs based on more efforts and a setback in the ramp-up process.

Overall, the KPIs are capable of representing the impact of the CCU to the rampup period of an assembly system. In case that $K_{\mathrm{PE}}$ and $K_{\mathrm{PQ}}$ point out the superiority of the cognitive interaction system, it is less cost-intensive and more flexible and robust than traditional automated systems. So an improvement of the ramp-up process has been reached and quantified. To prove these assumptions, several business and technology cases will be performed in the next funding period of the Cluster of Excellence while, up to now, the assembly cell (see Figure 4) and the CCU have been implemented and the four KPIs have been developed. These generated data will form the basis for supporting the decision making to apply cognitive systems in industry.

\section{Conclusion}

This paper proposes a set of KPIs which can determine the advantages of a cognitive interaction system in contrast to traditional automated systems to improve the ramp-up period of an assembly system. A precise cognitive interaction system in the domain of assembly planning systems is presented, which is the first self-optimising system in this domain. It comprises several cognitive capabilities implemented in the cognitive control unit (CCU) by a hybrid approach for assembly tasks, which enables robots to decide on their action during assembly autonomously.

To measure the systems' advantages involved, a set of key performance indicators is developed in this paper which can show the impact of this cognitive interaction system. These KPIs concentrate on the main improvements being achieved during the ramp-up phase of the assembly and its construction and sequence planning. The interaction of the four 
KPIs "planning effort," "acceleration of production volume growth," "increase of production volume," and "plan quality" evaluate the improvements in attaining the final production volume and in reducing the planning effort during ramp-up as well as the enhancement of the quality of the derived plan by means of the self-optimising capability of the CCU. These are developed in the context presented in this paper but designed to highlight the impact of cognitive interaction systems on production economics in general.

With respect to future research, there are plans to fill these KPIs with life, while in this paper the theoretical background for the next step has been set. Therefore, industrial applications are to be performed and analysed by comparing the assembly of a product including its ramp-up with the use of the CCU on the one hand and with the traditional approach on the other hand. Therein, possible weaknesses can be detected and resolved. This practical testing may then be shifted to other cognitive interaction systems to demonstrate the transferability of the set of KPIs. The challenge of fundamental research like the technological innovations developed within this Cluster of Excellence often comprises the persuasion of industry of the high performance of such solutions and the implementation or the launch of a product out of this. However, companies need reliable predictions on the applicability and economic efficiency. At this, the developed KPIs can play a major role as they provide exactly this required evidence in the examined topic.

\section{Acknowledgment}

The authors would like to thank the German Research Foundation DFG for supporting the research on cognitive technical systems including an economic analysis within the Cluster of Excellence "Integrative production technology for high-wage countries" at RWTH Aachen University.

\section{References}

[1] M. P. Mayer, C. M. Schlick, D. Ewert et al., "Automation of robotic assembly processes on the basis of an architecture of human cognition," Production Engineering, vol. 5, no. 4, pp. 423-431, 2011.

[2] A. A. Pufall, J. C. Fransoo, and A. G. kok, "What determines product ramp-up performance?: a review of characteristics based on a case study at Nokia mobile phones," BETA Publicatie: Working Paper 228, Technische Universiteit Eindhoven, 2007.

[3] H. Winkler, M. Heins, and P. Nyhuis, "A controlling system based on cause-effect relationships for the ramp-up of production systems," Production Engineering, vol. 1, no. 1, pp. 103-111, 2007.

[4] G. Schuh, W. Stölsle, and F. Straube, "Grundlagen des Anlaufmanagements: Entwicklungen und Trends, Definitionen und Begriffe, Integriertes Anlaufmanagementmodell," in Anlaufmanagement in der Automobilindustrie erfolgreich umsetzen, G. Schuh, Ed., pp. 1-6, Springer, Berlin, Germany, 2008.

[5] P. D. Ball, S. Roberts, A. Natalicchio, and C. Scorsafave, "Modelling production ramp-up of engineering products," Proceedings of the Institution of Mechanical Engineers B, vol. 225, no. 6, pp. 959971, 2011.

[6] R. Bischoff, Anlaufmanagement-Schnittstelle zwischen Projekt und Serie, vol. 2, Konstanser Managementschriften, 2007.

[7] D. Fitzek, Anlaufmanagement in Netzwerken: Grundlagen, Erfolgsfaktoren und Gestaltungsempfehlungen für die Automobilindustrie, Haupt, Bern, switzerland, 2006.

[8] K. Moeller, "Anlaufkosten in der Serienfertigung-Management und Controlling im Rahmen eines Lebenszyklus," in Synchronisation von Produktentwicklung und Produktionsprozess: ProduktreifeProduktneuanläufe Produktionsauslauf, H. Wildemann, Ed., TCW, 2005.

[9] J. Risse, Time-to-Market-Management in der Automobilindustrie: ein Gestaltungsrahmen für ein logistikorientiertes Anlaufmanagement, Haupt, Bern, Switzerland, 2003. 
[10] R. Schmitt and P. Beaujean, "Selbstoptimierende Produktionssysteme," Zeitschrift für wirtschaftliche Fabrikation, vol. 102, no. 9, pp. 520-524, 2007.

[11] E. Hauck, Ein kognitives Interaktionssystem zur Ansteuerung einer Montagezelle, vol. 812 of VDI Reihe 10, VDI, Düsseldorf, Germany, 2011.

[12] F. Klocke, "Production technology in high-wage countries-from ideas of today to products of tomorrow," Industrial Engineering and Ergonomics, vol. 1, pp. 13-30, 2009.

[13] C. Brecher, F. Klocke, R. Schmitt, and G. Schuh, Excellence in Production, Apprimus, Aachen, Germany, 2007.

[14] D. Ewert, S. Thelen, R. Kunze, M. Mayer, D. Schilberg, and S. Jeschke, "A graph based hybrid approach of offline pre-planning and online re-planning for efficient assembly under realtime constraints," in Proceedings of the International Conference on Intelligent Robotics and Applications (ICIRA '10), H. Liu, H. Ding, Z. Xiong, and X. Zhu, Eds., vol. 6425 of Part II, Lecture Notes in Computer Science, pp. 44-55, Springer, Shanghai, China, October 2010.

[15] E. Hauck, A. Gramatke, and K. Henning, "A software architecture for cognitive technical systems suitable for an assembly task in a production environment," in Automation Control-Theory and Practice, pp. 13-28, InTech, 2009.

[16] C. Buescher, M. Mayer, D. Schilberg, and S. Jeschke, "Artificial cognition in autonomous assembly planning systems," in Proceedings of the 4th International Conference Intelligent Robotics and Applications (ICIRA '11), J. Sabina, L. Honghai, and S. Daniel, Eds., vol. 7102 of Proceedings, Part II. Lecture Notes in Computer Science, pp. 168-178, Springer, Aachen, Germany, December 2011.

[17] T. Kempf, W. Herfs, and C. Brecher, "SOAR-based sequence control for a flexible assembly cell," in Proceedings of the IEEE Conference on Emerging Technologies and Factory Automation (ETFA '09), pp. 488496, IEEE Press, September 2009.

[18] M. A. Louly, A. Dolgui, and F. Hnaien, "Supply planning for single-level assembly system with stochastic component delivery times and service-level constraint," International Journal of Production Economics, vol. 115, no. 1, pp. 236-247, 2008.

[19] S. S. Chauhan, A. Dolgui, and J. M. Proth, "A continuous model for supply planning of assembly systems with stochastic component procurement times," International Journal of Production Economics, vol. 120, no. 2, pp. 411-417, 2009.

[20] J. Hoffmann, "FF: the fast-forward planning system," The AI Magazine, vol. 22, no. 3, pp. 57-62, 2001.

[21] C. Castellini, E. Giunchiglia, A. Tacchella, and O. Tacchella, "Improvements to satbased conformant planning," in Proceedings of the 6th European Conference on Planning (ECP '01), 2001.

[22] J. Hoffmann and R. Brafman, "Contingent planning via heuristic forward search with implicit belief states," in Proceedings of the 15th International Conference on Automated Planning and Scheduling (ICAPS '05), pp. 71-80, 2005.

[23] S. G. Kaufman, R. H. Wilson, and Calton, "Automated planning and programming of assembly of fully 3d mechanisms," Tech. Rep. SAND96-0433, Sandia National Laboratories, 1996.

[24] U. Thomas, Automatisierte Programmierung von Robotern für Montageaufgaben, Fortschritte in der Robotik 13, Shaker, Aachen, Germany, 2008.

[25] M. F. Zaeh and M. Wiesbeck, "A model for adaptively generating assembly instructions using statebased graph," in Manufacturing Systems and Technologies for the New Frontier, pp. 195-198, Springer, Berlin, Germany, 2008.

[26] M. Mayer, B. Odenthal, M. Faber, W. Kabuss, B. Kausch, and C. Schlick, "Simulation of human cognition in self-optimizing assembly systems," in Proceedings of the 17th World Congress on Ergonomics (IEA '09), 2009.

[27] L. S. Homem de Mello and A. C. Sanderson, "Representations of mechanical assembly sequences," IEEE Transactions on Robotics and Automation, vol. 7, no. 2, pp. 211-227, 1991.

[28] R. S. Chen, K. Y. Lu, and P. H. Tai, “Optimizing assembly planning through a three-stage integrated approach," International Journal of Production Economics, vol. 88, no. 3, pp. 243-256, 2004.

[29] P. E. Hart, N. J. Nilsson, and B. Raphael, "A formal basis for the heuristic determination of minimum cost paths," IEEE Transactions on Systems Science and Cybernetics, vol. 4, no. 2, pp. 100-107, 2007.

[30] P. Langley, J. E. Laird, and S. Rogers, "Cognitive architectures: research issues and challenges," Cognitive Systems Research, vol. 10, no. 2, pp. 141-160, 2009.

[31] D. Schilberg, Architektur eines Datenintegrators zur durchgängigen Kopplung von verteilten numerischen Simulationen [Dissertation], RWTH Aachen, Aachen, Germany, 2010.

[32] A. E. Gerevini, P. Haslum, D. Long, A. Saetti, and Y. Dimopoulos, "Deterministic planning in the fifth international planning competition: PDDL3 and experimental evaluation of the planners," Artificial Intelligence, vol. 173, no. 5-6, pp. 619-668, 2009. 


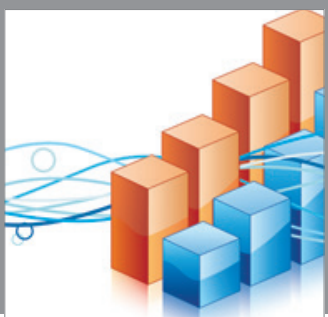

Advances in

Operations Research

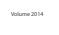

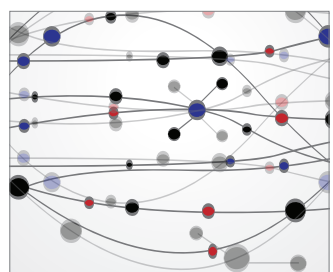

\section{The Scientific} World Journal
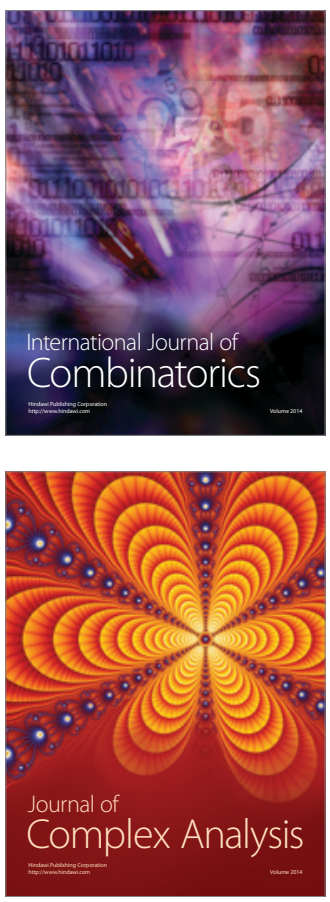

International Journal of

Mathematics and

Mathematical

Sciences
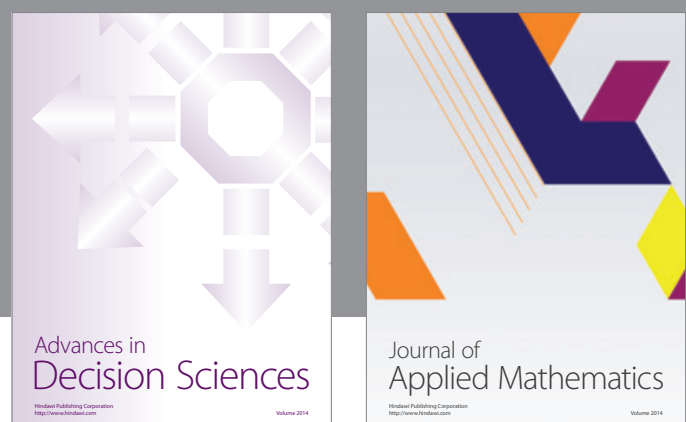

Journal of

Applied Mathematics
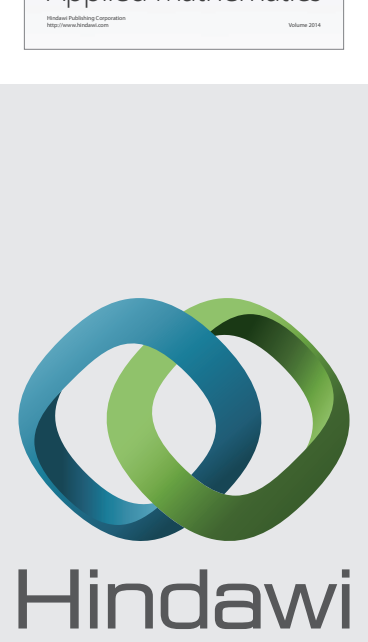

Submit your manuscripts at http://www.hindawi.com
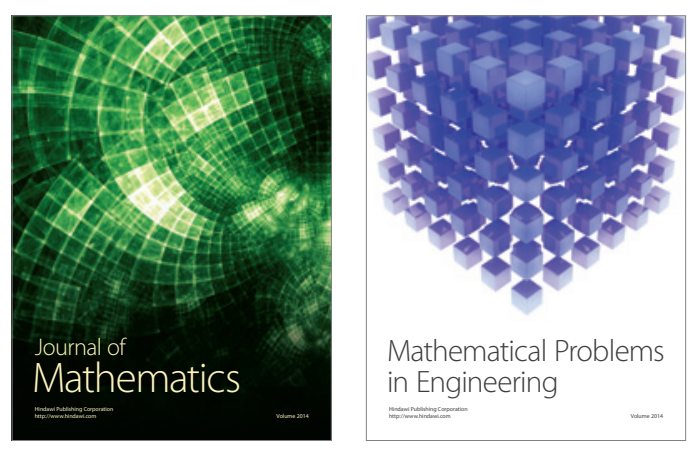

Mathematical Problems in Engineering
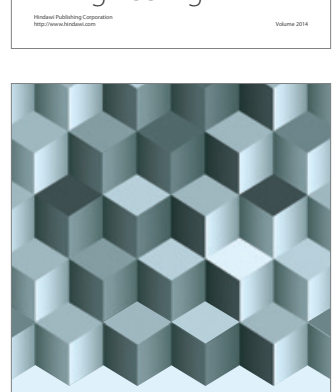

Journal of

Function Spaces
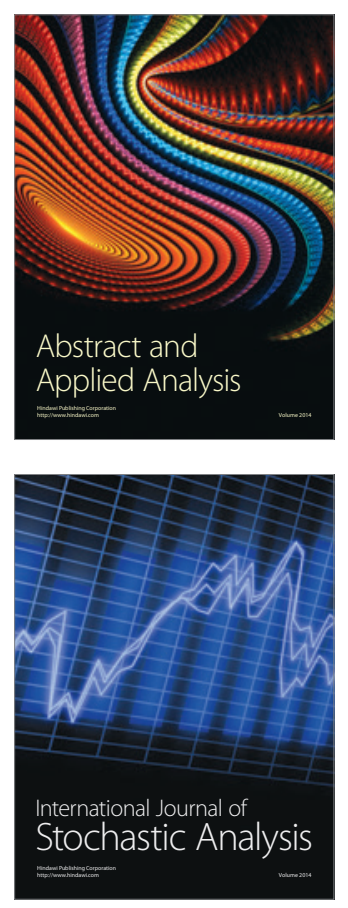

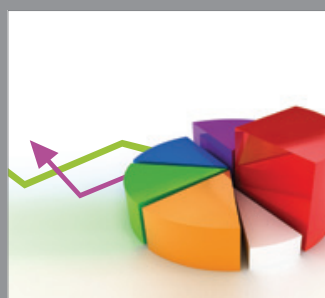

ournal of

Probability and Statistics

Promensencen
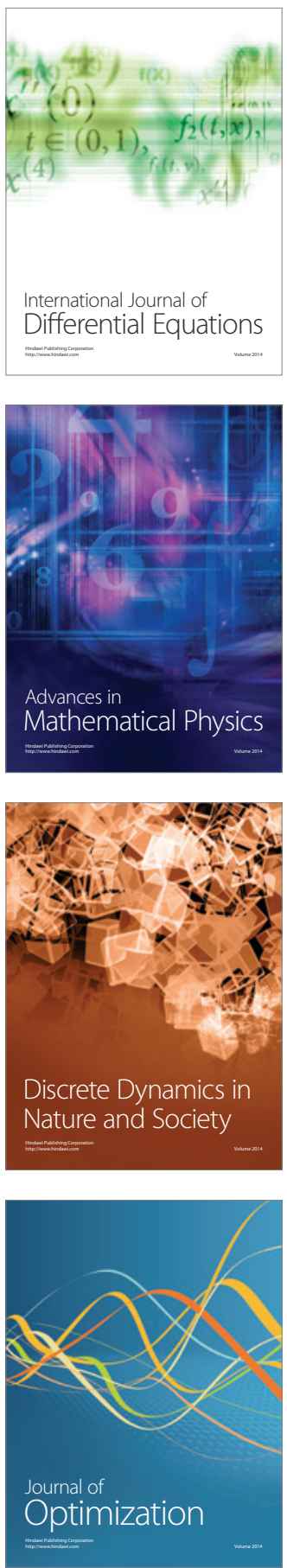